\title{
The Stable Set Polytope of Quasi-Line Graphs*
}

\author{
Friedrich Eisenbrand $^{\dagger} \quad$ Gianpaolo Oriolo $^{\star} \quad$ Gautier Stauffer ${ }^{\S} \quad$ Paolo Ventura $\mathbb{\text { II }}$
}

\begin{abstract}
It is a long standing open problem to find an explicit description of the stable set polytope of clawfree graphs. Yet more than 20 years after the discovery of a polynomial algorithm for the maximum stable set problem for claw-free graphs, there is even no conjecture at hand today.

Such a conjecture exists for the class of quasi-line graphs. This class of graphs is a proper superclass of line graphs and a proper subclass of claw-free graphs for which it is known that not all facets have 0/1 normal vectors. The Ben Rebea conjecture states that the stable set polytope of a quasi-line graph is completely described by clique-family inequalities. Chudnovsky and Seymour recently provided a decomposition result for claw-free graphs and proved that the Ben Rebea conjecture holds, if the quasi-line graph is not a fuzzy circular interval graph.

In this paper, we give a proof of the Ben Rebea conjecture by showing that it also holds for fuzzy circular interval graphs. Our result builds upon an algorithm of Bartholdi, Orlin and Ratliff which is concerned with integer programs defined by circular ones matrices.
\end{abstract}

\section{Introduction}

A graph $G$ is claw-free if no vertex has three pairwise nonadjacent neighbors. Line graphs are claw free and thus the weighted stable set problem for a claw-free graph is a generalization of the weighted matching problem of a graph. While the general stable set problem is NP-complete, it can be solved in polynomial time on a claw-free graph [22, 31] even in the weighted case [24, 25] see also [34]. These algorithms are extensions of Edmonds' $[11,10]$ matching algorithms.

The stable set polytope $\operatorname{STAB}(G)$ is the convex hull of the characteristic vectors of stable sets of the graph $G$. The polynomial equivalence of separation and optimization for rational polyhedra [17, $28,19]$ provides a polynomial time algorithm for the separation problem for $S T A B(G)$, if $G$ is clawfree. However, this algorithm is based on the ellipsoid method [20] and no explicit description of a set of inequalities is known that determines $\operatorname{STAB}(G)$ in this case. This apparent asymmetry between the algorithmic and the polyhedral status of the stable set problem in claw-free graphs gives rise to the challenging problem of providing a "... decent linear description of $S T A B(G)$ " [18], which is still open today. In spite of results characterizing the rank-facets [13] (facets with $0 / 1$ normal vectors) of claw-free graphs, or giving a compact lifted formulation for the subclass of distance claw-free graphs [29], the structure of the general facets for claw-free graphs is still not well understood and even no conjecture is at hand.

\footnotetext{
${ }^{*}$ An extended abstract of this work appeared in the proceedings of the Eleventh Conference on Integer Programming and Combinatorial Optimization, IPCO XI, 2005

${ }^{\dagger}$ Max-Planck-Institut für Informatik, Stuhlsatzenhausweg 85, D-66123 Saarbrücken, Germany, ei sen@mpi-sb . mpg • de

¥Universitá di Tor Vergata, Dipartimento di Ingegneria dell'Impresa, via del Politecnico 1, 00165, Roma, Italy, oriolo@disp.uniroma2.it

${ }^{\S}$ Ecole Polytechnique Fédérale de Lausanne, SB-IMA-ROSO Station 8, CH-1015 Lausanne, Switzerland, gautier.stauffer@epfl.ch

IIstituto di Analisi dei Sistemi ed Informatica - Antonio Ruberti del CNR, Viale Manzoni 30 - 00185 Roma, Italy, ventura@iasi.rm.cnr.it
} 
The matching problem [10] is a well known example of a combinatorial optimization problem in which the optimization problem on the one hand and the facets on the other hand are well understood. This polytope can be described by a system of inequalities in which the coefficients on the left-hand-side are $0 / 1$. This property of the matching polytope does not extend to the polytope $\operatorname{STAB}(G)$ associated with a claw-free graph. In fact, Giles and Trotter [15] show that for each positive integer $a$, there exists a claw-free graph $G$ such that $S T A B(G)$ has facets with $a /(a+1)$ normal vectors. Furthermore they show that there exist facets whose normal vectors have up to 3 different coefficients (indeed up to 5 as it is shown in [21]). Perhaps this is one of the reasons why providing a description of $\operatorname{STAB}(G)$ is not easy, since $0 / 1$ normal vectors can be interpreted as subsets of the set of nodes, whereas such an interpretation is not immediate if the normal vectors are not $0 / 1$.

A graph is quasi-line, if the neighborhood of any vertex partitions into two cliques. The complement of quasi-line graphs are called near-bipartite and an interesting polyhedral characterization of nearbipartite graphs is given in [23]; also a linear description of their stable set polytope has been given in [35]. The class of quasi-line graphs is a proper superclass of line graphs and a proper subclass of the class of claw-free graphs. Interestingly also for this class of graphs there are facets with $a /(a+1)$ normal vectors, for any nonnegative integer $a$ [15], but no facet whose normal vector has more than 2 different coefficients is known for this class.

\section{Clique family inequalities and the Ben Rebea conjecture}

We now describe the clique-family inequalities introduced in [27]. Our main result is a proof of the Ben Rebea conjecture, which essentially says that this proper generalization of the odd-set inequalities [10] which describe that matching polytope, together with the nonnegativity and clique inequalities, describe the stable set polytope of a quasi-line graph.

Let $\mathcal{F}=\left\{K_{1}, \ldots, K_{n}\right\}$ be a set of cliques, $1 \leq p \leq n$ be integral and $r=n \bmod p$. Let $V_{p-1} \subseteq V(G)$ be the set of vertices covered by exactly $(p-1)$ cliques of $\mathcal{F}$ and $V_{\geq p} \subseteq V(G)$ the set of vertices covered by $p$ or more cliques of $\mathcal{F}$. The inequality

$$
(p-r-1) \sum_{v \in V_{p-1}} x(v)+(p-r) \sum_{v \in V_{\geq p}} x(v) \leq(p-r)\left\lfloor\frac{n}{p}\right\rfloor
$$

is valid [27] for $\operatorname{STAB}(G)$ and is called the clique family inequality associated with $\mathcal{F}$ and $p$.

Clique family inequalities are a generalization of odd-set inequalities [10] which are part of the description of the matching polytope. This can be seen as follows. Suppose that the graph $G=(V(G), E(G))$ is the line graph of the graph $H=(V(H), E(H))$ and let $U \subseteq V(H)$ be an odd subset of the nodes of $H$.

The odd-set inequality defined by $U$ is the inequality

$$
\sum_{e \in E(U)} x(e) \leq\lfloor|U| / 2\rfloor
$$

which is valid for all characteristic vectors $\chi \in\{0,1\}^{E(H)}$ of matchings in $H$. Here, $E(U) \subseteq E(H)$ is the subset of edges of $H$ which have both endpoints in $U$.

This inequality is a clique-family inequality for the stable-set polytope of $G$, via the following construction. Each vertex $v \in U$ yields a clique $K_{v}$ in the line graph $G$ of $H$ consisting of the edges $e \in E(H)$, which are incident to $v$. The family of cliques $\mathcal{F}$ will consist of those cliques. Furthermore we let $p=2$. Since $|U|$ is odd the remainder $r$ is 1 . Furthermore, the vertices of $G$ which are in $V_{\geq p}$ are exactly the edges of $H$ which have both endpoints in $U \subseteq V(H)$. The clique family inequality corresponding to $\mathcal{F}$ and $p$ is therefore the odd-set inequality

$$
\sum_{v \in E(U)} x(v) \leq\lfloor|U| / 2\rfloor
$$


Ben Rebea [30] considered the problem to study $\operatorname{STAB}(G)$ for quasi-line graphs. Oriolo [27] formulated a conjecture inspired from his work.

Conjecture (Ben Rebea conjecture [27]). The stable set polytope of a quasi-line graph $G=(V, E)$ may be described by the following inequalities:

(i) $x(v) \geq 0$ for each $v \in V$

(ii) $\sum_{v \in K} x(v) \leq 1$ for each maximal clique $K$

(iii) inequalities (1) for each family $\mathcal{F}$ of maximal cliques and each integer $p$ with $|\mathcal{F}|>2 p \geq 4$ and $|\mathcal{F}| \bmod p \neq 0$.

In this paper we prove that the Ben Rebea Conjecture holds true. This is done by establishing the conjecture for fuzzy circular interval graphs, a class introduced by Chudnovsky and Seymour [6]. This settles the result, since Chudnovsky and Seymour showed that the conjecture holds if $G$ is quasi-line and not a fuzzy circular interval graph. Interestingly, since all the facets are rank for this latter class of graphs, the quasi-line graphs that "produce" non-rank facets are the fuzzy circular interval graphs.

We first show that we can focus our attention on circular interval graphs [6] a subclass of fuzzy circular interval graphs. The weighted stable set problem over a circular interval graph may be formulated as a packing problem $\max \left\{c x \mid A x \leq b, x \in \mathbb{Z}_{>0}^{n}\right\}$, where $b=\mathbf{1}$ and $A \in\{0,1\}^{m \times n}$ is a circular ones matrix, i.e., the columns of $A$ can be permuted in such a way that the ones in each row appear consecutively. Here the last and first entry of a row are also considered to be consecutive. Integer programs of this sort with general right-hand side $b \in \mathbb{Z}^{m}$ have been studied by Bartholdi, Orlin and Ratliff [3]. From this, we derive a separation algorithm which is based on the computation of a negative cycle, thereby extending a recent result of Gijswijt [14]. We then concentrate on packing problems with right-hand side $b=\alpha \mathbf{1}$, where $\alpha$ is an integer. By studying non-redundant cycles leading to separating hyperplanes, we show that each facet of the convex hull of integer feasible solutions to a packing problem of this sort has a normal vector with two consecutive coefficients. Instantiating this result with the case where $\alpha=1$, we obtain our main result.

\section{Cutting planes}

Before we proceed, we would like to stress some connections of this work to cutting plane theory. An inequality $c x \leq\lfloor\delta\rfloor$ is a Gomory-Chvátal cutting plane $[16,7]$ of a polyhedron $P \subseteq \mathbb{R}^{n}$, if $c \in \mathbb{Z}^{n}$ is an integral vector and $c x \leq \delta$ is valid for $P$. The Chvátal closure $P^{c}$ of $P$ is the intersection of $P$ with all its Gomory-Chvátal cutting planes. If $P$ is rational, then $P^{c}$ is a rational polyhedron [32]. The separation problem for $P^{c}$ is NP-hard [12]. A polytope $P$ has Chvátal-rank one, if its Chvátal closure is the integer hull $P_{I}$ of $P$. Let $Q S T A B(G)$ be the fractional stable set polytope of a graph $G$, i.e., the polytope defined by non-negativity and clique inequalities. A famous example of a polytope of Chvátal-rank one is the fractional matching polytope and thus $\operatorname{QSTAB}(G)$, where $G$ is a line graph. Giles and Trotter [15] showed that the Chvátal rank of $Q S T A B(G)$ is at least two, if $G$ is claw-free. Chvátal, Cook and Hartman [8] showed that the Chvátal-rank of $\operatorname{QSTAB}(G)$ grows logarithmically in the number of nodes, even if the stability number of $G$ is two and thus, even if $G$ is claw-free. Oriolo [27] has shown that $\operatorname{QSTAB}(G)$ has Chvátal rank at least two, if $G$ is a quasi-line graph.

An inequality $c x \leq \delta$ is called a split cut [9] of $P$ if there exists an integer vector $\pi \in \mathbb{Z}^{n}$ and an integer $\pi_{0}$ such that $c x \leq \delta$ is valid for $P \cap\left\{x \in \mathbb{R}^{n} \mid \pi x \leq \pi_{0}\right\}$ and for $P \cap\left\{x \in \mathbb{R}^{n} \mid \pi x \geq \pi_{0}+1\right\}$. The split closure $P^{s}$ of $P$ is the intersection of $P$ with all its split cuts and this is a rational polyhedron if $P$ itself is rational [9, 2]. The separation problem for the split closure is also NP-hard [4]. A polyhedron $P \subseteq \mathbb{R}^{n}$ has split-rank one, if $P^{s}=P_{I}$. Since a Gomory-Chvátal cutting plane is also a split cut one has $P^{s} \subseteq P^{c}$. 
Both cutting plane calculi are simple procedures to derive valid inequalities for the integer hull of a polyhedron. We show below that a clique family inequality is a split cut for $\operatorname{QSTAB}(G)$ with $\pi(v)=1$ if $v \in V_{p-1} \cup V_{\geq p}, \pi(v)=0$ otherwise and $\pi_{0}=\left\lfloor\frac{n}{p}\right\rfloor$. Thus, while the fractional stable set polytope of a quasi-line graph does not have Chvátal rank one, its split-rank is indeed one.

In the remainder of this section, we present the split-cut derivation of the clique-family inequality. Notice that the inequality

$$
(p-1) \sum_{v \in V_{p-1}} x(v)+p \sum_{v \in V_{\geq p}} x(v) \leq n=p\lfloor n / p\rfloor+r
$$

is valid for $\operatorname{QSTAB}(G)$, since it is the result of summing up the clique inequalities corresponding to $\mathcal{F}$ and possibly applying the lower bounds $-x(v) \leq 0$ on vertices $v \in V_{\geq p}$ which are contained in more than $p$ cliques. Now consider the disjunction

$$
\sum_{v \in V_{p-1} \cup V_{\geq p}} x(v) \leq\lfloor n / p\rfloor \quad \vee \quad \sum_{v \in V_{p-1} \cup V_{\geq p}} x(v) \geq\lfloor n / p\rfloor+1
$$

Assume now the left inequality of the disjunction (5). Under this assumption we can write

$$
\begin{aligned}
(p-r-1) \sum_{v \in V_{p-1}} x(v)+(p-r) \sum_{v \in V_{\geq p}} x(v) & \leq(p-r) \sum_{v \in V_{p-1} \cup V_{\geq p}} x(v) \\
& \leq(p-r)\lfloor n / p\rfloor,
\end{aligned}
$$

where the first inequality follows from the lower bounds on the variables.

Assume now the right inequality of the disjunction (5). Together with (4) we can write

$$
\begin{aligned}
(p-r-1) & \sum_{v \in V_{p-1}} x(v)+(p-r) \sum_{v \in V_{\geq p}} x(v) \\
& =(p-1) \sum_{v \in V_{p-1}} x(v)+p \sum_{v \in V_{\geq p}} x(v)-r \sum_{v \in V_{p-1} \cup V_{\geq p}} x(v) \\
& \leq(p-r)\lfloor n / p\rfloor .
\end{aligned}
$$

\section{From quasi-line graphs to circular interval graphs}

In this section we first review some results concerning the structure of quasi-line graphs due to Chudnovsky and Seymour [6]. We then build upon these results to reduce the proof of the Ben Rebea conjecture to the case where the graph is a circular interval graph.

\subsection{Circular Interval Graphs}

A circular interval graph [6] $G=(V, E)$ is defined by the following construction, see Figure 1: Take a circle $C$ and a set of vertices $V$ on the circle. Take a subset of intervals $I$ of $C$ and say that $u, v \in V$ are adjacent if $u$ and $v$ are contained in one of the intervals.

Any interval used in the construction will correspond to a clique of $G$. Denote the family of cliques stemming from intervals by $\mathcal{K}_{I}$ and the set of all cliques in $G$ by $K(G)$. Without loss of generality, the (intervals) cliques of $\mathcal{K}_{I}$ are such that none includes another. Moreover $\mathcal{K}_{I} \subseteq K(G)$ and each edge of $G$ is contained in a clique of $\mathcal{K}_{I}$. Therefore, if we let $A \in\{0,1\}^{m \times n}$ be the clique vertex incidence matrix of $\mathcal{K}_{I}$ and $V$ one can formulate the (weighted) stable set problem on a circular interval graph as a packing problem

$$
\begin{aligned}
\max \quad \sum_{v \in V} c(v) x(v) & \\
A x & \leq \mathbf{1} \\
x(v) & \in\{0,1\} \quad \forall v \in V
\end{aligned}
$$

where the matrix $A$ is a circular ones matrix (e.g. using clockwise ordering of the vertices). 

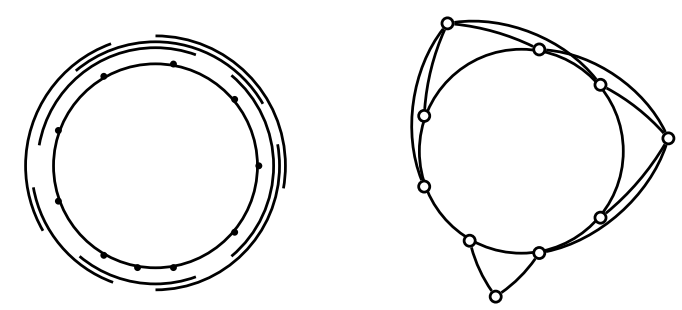

Figure 1: A circular interval graph

We point out that the property above may be used as a characterization for circular interval graphs. In fact, it is easy to see that a graph $G(V, E)$ is a circular interval graph if and only if there exists an ordering of $V$ and a set $\mathcal{K}_{I}$ of cliques of $G$ such that: $(i)$ each edge of $G$ is contained in a clique of $\mathcal{K}_{I}$; (ii) the clique vertex incidence matrix of $\mathcal{K}_{I}$ and $V$ is a circular ones matrix. Finally, circular interval graphs are also called proper circular arc graphs, i.e. they are equivalent to the intersection graphs of arcs of a circle with no containment between arcs [6].

\subsection{Fuzzy Circular Interval Graphs}

Chudnovsky and Seymour [6] also introduced a super-class of circular interval graphs called fuzzy circular interval graphs. A graph $G(V, E)$ is a fuzzy circular interval if the following conditions hold.

(i) There is a map $\Phi$ from $V$ to a circle $\mathcal{C}$.

(ii) There is a set of intervals $I$ of $\mathcal{C}$, none including another, such that no point of $\mathcal{C}$ is an endpoint of more than one interval so that:

(a) If two vertices $u$ and $v$ are adjacent, then $\Phi(u)$ and $\Phi(v)$ belong to a common interval.

(b) If two vertices $u$ and $v$ belong to a same interval, which is not an interval with endpoints $\Phi(u)$ and $\Phi(v)$, then they are adjacent.

In other words, in a fuzzy circular interval graph, adjacencies are completely described by the pair $(\Phi, I)$, except for vertices $u$ and $v$ such that $I$ contains an interval with endpoints $\Phi(u)$ and $\Phi(v)$. For these vertices adjacency is fuzzy. If $[p, q]$ is an interval of $I$ such that $\Phi^{-1}(p)$ and $\Phi^{-1}(q)$ are both non-empty, then we call the cliques $\left(\Phi^{-1}(p), \Phi^{-1}(q)\right)$ a fuzzy pair. Here $\Phi^{-1}(p)$ denotes the clique $\{v \in V \mid \Phi(v)=p\}$.

The left drawing of Figure 2 illustrates a section of a representation of a fuzzy circular interval graph. The cliques $\Phi^{-1}(p)$ and $\Phi^{-1}(r)$ are fuzzy pairs, since $p$ and $r$ are the endpoints of an interval. The node sets $\Phi^{-1}(p) \cup \Phi^{-1}(q)$ and $\Phi^{-1}(q) \cup \Phi^{-1}(r)$ are cliques. The edges with one endpoint in $\Phi^{-1}(p)$ and the other in $\Phi^{-1}(r)$ are "fuzzy". The other interval which starts a little left from $q$ and ends at $s$ can be extended a little to the right of $s$, since $\Phi^{-1}(q) \cup \Phi^{-1}(r) \cup \Phi^{-1}(s)$ is a clique of $G$. Therefore the right drawing of Figure 2 shows another possible representation of the same graph.
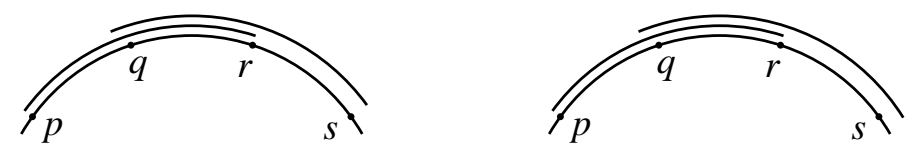

Figure 2: Two different representations of a fuzzy circular interval graph 
Trivially, if a fuzzy circular interval graph admits a fuzzy representation $(\Phi, I)$ with no fuzzy pairs of cliques, then $G$ is a circular interval graph. We can sharpen this latter statement if we recall the definition of homogeneous pair of cliques.

Two disjoint cliques $K_{1}$ and $K_{2}$ of size at least two are a homogeneous pair of cliques [6] if no vertex $v \notin K_{1} \cup K_{2}$ is both a neighbor and a non-neighbor of a vertex of $K_{1}$ or both a neighbor and a non-neighbor of a vertex of $K_{2}$ respectively. Trivially, if $[p, q]$ is an interval of $I$ such that $\Phi^{-1}(p)$ and $\Phi^{-1}(q)$ are both of size at least two, then $\Phi^{-1}(p)$ and $\Phi^{-1}(q)$ is a homogeneous pair.

We also say that a homogeneous pair of cliques $\left(K_{1}, K_{2}\right)$ is proper if every vertex of $K_{1}$ has a neighbor and a non-neighbor in $K_{2}$ and every vertex of $K_{2}$ has a neighbor and a non-neighbor in $K_{1}$. A graph is $C_{4}$-free if it does not have an induced subgraph isomorphic to a cordless cycle of length 4 . For $X \subseteq V$, we denote by $G[X]$ the subgraph of $G$ induced by $X$.

Lemma 1. [5] Let $\left(K_{1}, K_{2}\right)$ be a homogeneous pair of cliques. If $\left(K_{1}, K_{2}\right)$ is proper, then the subgraph $G\left[K_{1} \cup K_{2}\right]$ contains an induced $C_{4}$.

Proof. For a vertex $u \in K_{1}$ let $d_{2}(u)$ be its degree with respect to $K_{2}$, that is $d_{2}(u)=\left|\left\{v \in K_{2}: u v \in E\right\}\right|$. Let $u_{1}$ be a vertex of $K_{1}$ with maximum degree with respect to $K_{2}$. Since $\left(K_{1}, K_{2}\right)$ is proper, $u_{1}$ has a non-neighbor $z_{2}$ in $K_{2}$. The same applies to $z_{2}$ and $K_{1}: z_{2}$ has a neighbor $u_{2} \in K_{1}$. Finally, there must exist a vertex $z_{1} \in K_{2}$ that is a neighbor of $u_{1}$ and a non-neighbor of $u_{2}$ (otherwise $d_{2}\left(u_{2}\right)>d_{2}\left(u_{1}\right)$ ). It follows that $\left\{u_{1}, u_{2}, z_{1}, z_{2}\right\}$ induce a $C_{4}$.

Lemma 2. Let $G$ be a fuzzy circular interval graph with a representation $(\Phi, I)$. If no fuzzy pair contains an induced $C_{4}$, then $G$ is a circular interval graph.

Proof. Let $\left(\Phi^{-1}(p), \Phi^{-1}(q)\right)$ be a fuzzy pair. This pair is homogenous but not proper by Lemma 1 . Since it is not proper, there exists a vertex in $\Phi^{-1}(p)$ (resp. $\Phi^{-1}(q)$ ) that is either totally joined to or independent from $\Phi^{-1}(q)$ (resp. $\Phi^{-1}(p)$ ).

Suppose that $v \in \Phi^{-1}(p)$ is totally joined to $\Phi^{-1}(q)$. Then we can move $\Phi(v)$ by a small amount into the interior of the interval $[p, q]$. This yields a new representation $\left(\Phi^{\prime}, I\right)$ of the graph $G$ that does not introduce new fuzzy pairs and reduces the number of vertices which are contained in a fuzzy pair by one.

Similarly, if $v \in \Phi^{-1}(p)$ is independent from $\Phi^{-1}(q)$, we can move $\Phi(v)$ such that it is outside $[p, q]$. This operation yields a new mapping $\Phi^{\prime}$. In addition to that we must add an interval $I$ covering $v$ and its neighbors in $[p, q]$. Since $v$ is connected to every vertex which is mapped to the half-open interval $[p, q)$ and since $v \cup \Phi^{\prime-1}([p, q))$ is a clique, this interval $I$ can be chosen such that both of its endpoints are not contained in $\Phi^{\prime}(V)$. This new representation $\left(\Phi^{\prime}, I \cup\{I\}\right)$ does also not introduce new fuzzy pairs and reduces the number of vertices which are contained in a fuzzy pair by one.

We can iterate this process until there are no fuzzy pairs left.

\subsection{Decomposition of quasi-line graphs}

Let $G$ be a graph and $L(G)$ be its line graph. Notice that $G$ can be build by considering a disjoint union of stars (associated to every vertex in $G$ ) and then identifying some of the edges. $L(G)$ can thus be built by considering a disjoint union of cliques and identifying some vertices. This construction has been generalized by Chudnovsky and Seymour [6] through the operations glue and composition.

A vertex $v$ is simplicial if its neighbors form a clique. A strip $(G, a, b)$ is a graph $G$ together with two designated simplicial vertices $a$ and $b$. Let $(G, a, b)$ and $\left(G^{\prime}, a^{\prime}, b^{\prime}\right)$ be two vertex-disjoint strips. The glue of $(G, a, b)$ and $\left(G^{\prime}, a^{\prime}, b^{\prime}\right)$ is the graph resulting from the union of $G \backslash\{a, b\}$ and $G^{\prime} \backslash\left\{a^{\prime}, b^{\prime}\right\}$ together with the adjunction of all possible edges between the neighbors of $a(b)$ and the neighbors of $a^{\prime}\left(b^{\prime}\right)$. 
Let $G_{0}$ be a disjoint union of cliques with an even vertex set $V\left(G_{0}\right)=\left\{a_{1}, b_{1}, \ldots, a_{n}, b_{n}\right\}$. Let $\left(G_{i}^{\prime}, a_{i}^{\prime}, b_{i}^{\prime}\right)$ be $n$ strips that are vertex-disjoint, also from $G_{0}$. For $i=1, \ldots, n$, let $G_{i}$ be the graph obtained by gluing $\left(G_{i-1}, a_{i}, b_{i}\right)$ with $\left(G_{i}^{\prime}, a_{i}^{\prime}, b_{i}^{\prime}\right) . G_{n}$ is called the composition of the strips $\left(G_{i}^{\prime}, a_{i}^{\prime}, b_{i}^{\prime}\right)$, with the collection of disjoint cliques $G_{0}$.

We observed that a line graph can be built by considering a disjoint union of cliques and identifying some vertices. Gluing a 2-edges strip with a strip $(S, a, b)$ results in the identification of $a$ and $b$. Therefore any line graph $G$ can be expressed as the composition of 2-edges strips with $G_{0}$ made of $|V(G)|$ cliques. If we now replace 2-edges strips by fuzzy linear interval strips (a superclass), we get a large class of quasi-line graphs. Chudnovsky and Seymour proved in fact the following structural result.

Theorem 3 (Chudnovsky and Seymour [6]). A connected quasi-line graph $G$ is either a fuzzy circular interval graph, or it is the composition of fuzzy linear interval strips with a collection of disjoint cliques.

Chudnovsky and Seymour were also able to give a complete characterization of the stable set polytope of quasi-line graphs that are not fuzzy circular interval graphs. Let $\mathcal{F}=\left\{K_{1}, K_{2}, \ldots, K_{2 n+1}\right\}$ be an odd set of cliques of $G$. Let $T \subseteq V$ be the set of vertices which are covered by at least two cliques of $\mathcal{F}$. Then the inequality $\sum_{v \in T} x(v) \leq n$ is a valid inequality for $\operatorname{STAB}(G)$ and inequalities of this type are called Edmonds inequalities.

Theorem 4 (Chudnovsky and Seymour [6]). If $G$ is the composition of fuzzy linear interval strips with a collection of disjoint cliques, then all non trivial facets of $S T A B(G)$ are Edmonds inequalities.

\subsection{The reduction to circular interval graphs}

Observe that Edmonds inequalities are special clique family inequalities associated with $\mathcal{F}$ and $p=2$. Therefore, we may give a positive answer to the Ben Rebea Conjecture if we prove that it holds for fuzzy circular interval graphs. We now show that it will be enough to prove the conjecture for circular interval graphs.

Lemma 5. Let $F$ be a facet of $\operatorname{STAB}(G)$, where $G$ is a fuzzy circular interval graph. Then $F$ is also a facet of $\operatorname{STAB}\left(G^{\prime}\right)$, where $G^{\prime}$ is a circular interval graph and is obtained from $G$ by removing some edges.

Proof. Suppose that $F$ is induced by the valid inequality $a x \leq \beta$. An edge $e$ is $F$-critical, if $a x \leq \beta$ is not valid for $\operatorname{STAB}(G \backslash e)$. If $e$ is not $F$-critical, then $F$ is also a facet of $\operatorname{STAB}(G \backslash e)$.

Let $(\Phi, I)$ be a fuzzy representation of $G$. For every fuzzy pair $\left(K_{1}, K_{2}\right)$, we remove all the edges connecting a vertex in $K_{1}$ to a vertex in $K_{2}$ that are non $F$-critical. We end up with a fuzzy circular interval graph $G^{\prime}$ which has the same fuzzy representation $(\Phi, I)$ as $G$.

We claim that no fuzzy pair of $G^{\prime}$ contains a $C_{4}$ and thus by Lemma 2, $G^{\prime}$ is a circular interval graph. Moreover since we remove only non $F$-critical edges, $F$ is still a facet of $\operatorname{STAB}\left(G^{\prime}\right)$.

Suppose the contrary that there exists a fuzzy pair $\left(K_{1}, K_{2}\right)$ of $G^{\prime}$ that contains a $C_{4}$. Say $V\left(C_{4}\right)=$ $\left\{u_{1}, u_{2}, v_{1}, v_{2}\right\}$ with $u_{1}, u_{2} \in K_{1}, v_{1}, v_{2} \in K_{2}, u_{1} v_{1}, u_{2} v_{2} \in E\left(C_{4}\right)$. The edge $u_{1} v_{1}$ is $F$-critical. Hence there exists a stable set $S$ containing $u_{1}$ and $v_{1}$ such that $S$ violates $a x \leq \beta$ and $S$ is stable in $G^{\prime} \backslash u_{1} v_{1}$. Since $K_{1}$ and $K_{2}$ form an homogeneous pair, $u_{1}$ and $u_{2}$ are adjacent to the same vertices in $G^{\prime} \backslash K_{2}$. This implies that $\left(S \backslash u_{1}\right) \cup u_{2}$ is a stable set. Therefore $a\left(u_{2}\right)<a\left(u_{1}\right)$ (else $\left(u_{1}, v_{1}\right)$ is not $F$-critical). Applying the same argument to $u_{2} v_{2}$ leads to $a\left(u_{1}\right)<a\left(u_{2}\right)$. Which is a contradiction.

Remark. We would like to point out here that the following statement can be proved in a similar way as the proof of Lemma 5: Let $F$ be a facet of $S T A B(G)$. There exists a graph $G^{\prime}$, obtained from $G$ by removing some edges, such that $F$ is also a facet of $S T A B\left(G^{\prime}\right)$ and $G^{\prime}$ does not contain any pair of cliques which is proper and homogeneous. 
Lemma 5 shows that each facet of a fuzzy circular interval graph is a facet of a circular interval graph which is obtained via the deletion of some edges. A clique family inequality of the thereby obtained circular interval graph is a clique family inequality of the original fuzzy circular interval graph. Therefore, we now only have to establish the Ben Rebea conjecture for the class of circular interval graphs. Recall that the stable set polytope of a circular interval graph is the integer hull of a polyhedron of the form $\left\{x \in \mathbb{R}^{n} \mid A x \leq 1, x \geq 0\right\}$, where $A \in\{0,1\}^{m \times n}$ is a circular ones matrix.

\section{Slicing and separation}

In this section we show that the separation problem for $\operatorname{STAB}(G)$ reduces to a min-cost circulation problem if $G$ is a circular interval graph. For this, we present a membership algorithm of Gijswijt [14] and develop it further to retrieve a separating hyperplane.

Let $P$ be a polytope $P=\left\{x \in \mathbb{R}^{n} \mid A x \leq b, x \geq 0\right\}$, where $A \in\{0,1\}^{m \times n}$ is a circular ones matrix and $b \in \mathbb{Z}^{m}$ an integral vector. We consider the separation problem for the integer hull $P_{I}$ of $P$ :

Given $x^{*} \in \mathbb{R}^{n}$, determine, whether $x^{*} \in P_{I}$ and if not, determine an inequality $c x \leq \delta$ which is valid for $P_{I}$ and satisfies $c x^{*}>\delta$.

Following Bartholdi, Orlin and Ratliff [3], we consider the unimodular transformation $x=T y$, where $T$ is the unimodular matrix

$$
T=\left(\begin{array}{ccccccc}
1 & & & & & \\
-1 & 1 & & & & \\
& -1 & 1 & & & \\
& & -1 & & & \\
& & & \ddots & & \\
& & & & 1 & \\
& & & & & -1 & 1
\end{array}\right)
$$

The problem then reads, separate $y^{*}=T^{-1} x^{*}$ from the integer hull $Q_{I}$ of the polytope $Q$ defined by the system

$$
\left(\begin{array}{c}
A \\
-I
\end{array}\right) T y \leq\left(\begin{array}{l}
b \\
0
\end{array}\right)
$$

In the following we denote the inequality system (7) by $B y \leq d$. Let us rewrite the matrix $B$ as $B=(N \mid v)$, i.e. $v$ is the $n$-th column of $B$. Observe that, by construction, $v$ is also the last column of $\left(\begin{array}{c}A \\ -I\end{array}\right)$.

Each row of the matrix $N$ has at most one entry which is +1 and at most one entry which is -1 . All other entries are 0 . The matrix $N$ is thus totally unimodular. Thus, whenever $y(n)$ is set to an integer $\beta \in \mathbb{Z}$, the possible values for the variables $y(1), \ldots, y(n-1)$ define an integral polytope $Q_{\beta}=Q \cap\{y \in$ $\left.\mathbb{R}^{n} \mid y(n)=\beta\right\}$. We call this polytope $Q_{\beta}$ the slice of $Q$ defined by $\beta$.

Since $T$ is unimodular, the corresponding slice of the original polyhedron $P \cap\left\{x \in \mathbb{R}^{n} \mid \sum_{i=1}^{n} x(i)=\beta\right\}$ is an integral polyhedron. From this it is already easy to see that the split-rank of $P$ is one. However, we present a combinatorial separation procedure for the integer hull $P_{I}$ of $P$ which computes a split cut via the computation of a negative cycle.

If $y^{*}(n)$ is integral, then $y^{*}$ lies in $Q_{I}$ if and only if $y^{*} \in Q_{y^{*}(n)}$. Therefore we assume in the following that $y^{*}(n)$ is not integral and let $\beta$ be an integer such that $\beta<y^{*}(n)<\beta+1$ and let $1>\mu>0$ be the real number with $y^{*}(n)=\beta+1-\mu$. Furthermore, let $Q_{L}$ and $Q_{R}$ be the left slice $Q_{\beta}$ and right slice $Q_{\beta+1}$ respectively. A proof of the next lemma follows from basic convexity.

Lemma 6. The point $y^{*}$ lies in $Q_{I}$ if and only if there exist $y_{L} \in Q_{L}$ and $y_{R} \in Q_{R}$ such that

$$
y^{*}=\mu y_{L}+(1-\mu) y_{R}
$$


In the following we denote by $\bar{y} \in \mathbb{R}^{n-1}$ the vector of the first $n-1$ components of $y \in \mathbb{R}^{n}$. From the above discussion one has $y^{*} \in Q_{I}$ if and only if the following linear constraints have a feasible solution.

$$
\begin{aligned}
\overline{y_{L}}+\overline{y_{R}} & =\overline{y^{*}} \\
N \overline{y_{L}} & \leq \mu d_{L} \\
N \overline{y_{R}} & \leq(1-\mu) d_{R}
\end{aligned},
$$

where $d_{L}=d-\beta v$ and $d_{R}=d-(\beta+1) v$.

Using Farkas' Lemma [33], it follows that the system (8) is feasible, if and only if $\sum_{i=1}^{n-1} \lambda(i) y^{*}(i)+$ $\mu f_{L} d_{L}+(1-\mu) f_{R} d_{R}$ is nonnegative, whenever $\lambda, f_{L}$ and $f_{R}$ satisfy

$$
\begin{aligned}
\lambda+f_{L} N & =0 \\
\lambda+f_{R} N & =0 \\
f_{L}, f_{R} & \geq 0 .
\end{aligned}
$$

Now $\lambda+f_{L} N=0$ and $\lambda+f_{R} N=0$ is equivalent to $\lambda=-f_{L} N$ and $f_{L} N=f_{R} N$. Thus (8) defines a feasible system, if and only if the optimum value of the following linear program is nonnegative

$$
\begin{aligned}
\min -f_{L} N \overline{y^{*}}+\mu f_{L} d_{L}+(1-\mu) f_{R} d_{R} \\
f_{L} N=f_{R} N \\
f_{L}, f_{R} \geq 0 .
\end{aligned}
$$

Let $w$ be the negative sum of the columns of $N$. Then (10) is the problem of finding a minimum cost circulation in the directed graph $D=(U, \mathcal{A})$ defined by the edge-node incidence matrix

$$
M=\left(\begin{array}{cc}
N & w \\
-N & -w
\end{array}\right) \text { and edge weights } \mu\left(-N \overline{y^{*}}+d_{L}\right),(1-\mu)\left(-N \overline{y^{*}}+d_{R}\right)
$$

Thus $y^{*} \notin Q_{I}$ if and only if there exists a negative cycle in $D=(U, \mathcal{A})$. The membership problem for $Q_{I}$ thus reduces to the problem of detecting a negative cycle in $D$, see [14].

A separating split cut for $y^{*}$ is an inequality which is valid for $Q_{L}$ and $Q_{R}$ but not valid for $y^{*}$. The inequality $f_{L} N \bar{y} \leq f_{L} d_{L}$ is valid for $Q_{L}$ and the inequality $f_{R} N \bar{y} \leq f_{R} d_{R}$ is valid for $Q_{R}$. The corresponding disjunctive inequality (see, e.g., [26]) is the inequality

$$
f_{L} N \bar{y}+c(n) y(n) \leq \delta \text {, where } c(n)=f_{L} d_{L}-f_{R} d_{R} \text { and } \delta=(\beta+1) f_{L} d_{L}-\beta f_{R} d_{R} .
$$

The polytopes $Q_{L}$ and $Q_{R}$ are defined by the systems

$$
\begin{aligned}
y(n) & =\beta \\
N \bar{y}+v y(n) & \leq d
\end{aligned} \quad \text { and } \quad \begin{aligned}
y(n) & =\beta+1 \\
N \bar{y}+v y(n) & \leq d
\end{aligned}
$$

respectively.

Let $f_{L, 0}$ be the number $c(n)-f_{L} v$. Then the inequality (12) can be derived from the system defining $Q_{L}$ with the weights $\left(f_{L, 0}, f_{L}\right)$. Notice that, if $y^{*}$ can be separated from $Q_{I}$, then $f_{L, 0}$ must be positive. This is because $y^{*}$ violates (12) and satisfies the constraints (13) on the left, where the equality $y(n)=\beta$ in the first line is replaced with $y(n) \geq \beta$. Let $f_{R, 0}$ be the number $c(n)-f_{R} v$. Then the inequality (12) can be derived from the system defining $Q_{R}$ with the weights $\left(f_{R, 0}, f_{R}\right)$. Notice that, if $y^{*}$ can be separated from $Q_{I}$, then $f_{R, 0}$ must be negative.

A negative cycle in a graph with $m$ edges and $n$ nodes can be found in time $O(m n)$, see, e.g. [1]. Translated back to the original space and to the polyhedron $P$ this gives the following theorem. 
Theorem 7. The separation problem for $P_{I}$ can be solved in time $O(m n)$. Moreover, if $x^{*} \in P$ and $x^{*} \notin P_{I}$ one can compute in $O(m n)$ a split cut $c x \leq \delta$ which is valid for $P_{I}$ and separates $x^{*}$ from $P_{I}$ together with a negative integer $f_{R, 0}$, a positive integer $f_{L, 0}$ and a vector $f_{L}, f_{R}$, which is the incidence vector of a simple negative cycle of the directed graph $D=(U, \mathcal{A})$ with edge-node incidence matrix and weights as in (11), such that $c x \leq \delta$ is derived with from the systems

$$
\begin{aligned}
& \mathbf{1} x \leq \beta \quad-\mathbf{1} x \leq-(\beta+1) \\
& A x \leq b \quad \text { and } \quad A x \leq b \\
& -x \leq 0 . \quad-x \leq 0,
\end{aligned}
$$

with the weights $f_{L, 0}, f_{L}$ and $\left|f_{R, 0}\right|, f_{R}$ respectively.

The above theorem gives an explicit derivation of the separating hyperplane as a split cut of $P$. We have the following corollary.

Corollary 8. The integer hull $P_{I}$ is the split closure of $P$.

\section{The facets of $P_{I}$ for the case $b=\alpha \cdot 1$}

In this section we study the facets of $P_{I}$, where $P=\left\{x \in \mathbb{R}^{n} \mid A x \leq b, x \geq 0\right\}$, where $A$ is a circular ones matrix and $b$ is an integer vector of the form $\alpha \mathbf{1}, \alpha \in \mathbb{N}$. For this, we actually inspect how the facets of the transformed polytope $Q$ described in Section 3 are derived from the systems (13) and apply this derivation to the original system. It will turn out that the facet normal-vectors of $P_{I}$ have only two integer coefficients, which are in addition consecutive. Since the stable set polytope of a circular interval graph is defined by such a system with $\alpha=1$, we can later instantiate the results of this section to this special case. We can assume that the rows of $A$ are inclusion-wise maximal.

Let $F$ be a facet of $Q_{I}$ and let $y^{*}$ be in the relative interior of $F$. This facet $F$ is generated by the unique inequality (12), which corresponds to a simple cycle of (10) of weight 0 . Furthermore assume that $F$ is not induced by an inequality $y(n) \leq \gamma$ for some $\gamma \in \mathbb{Z}$. Since $F$ is a facet of the convex hull of integer points of two consecutive slices, we can assume that $y^{*}(n)=\beta+1 / 2$ and thus that $\mu=1 / 2$ in (10). This allows us to rewrite the objective function of problem (10) as follows:

$$
\min \left(s^{*}+\frac{1}{2} v\right) f_{L}+\left(s^{*}-\frac{1}{2} v\right) f_{R}
$$

where $s^{*}$ is the slack vector

$$
s^{*}=\left(\begin{array}{c}
\alpha \mathbf{1} \\
\mathbf{0}
\end{array}\right)-B y^{*}=\left(\begin{array}{c}
\alpha \mathbf{1} \\
\mathbf{0}
\end{array}\right)-\left(\begin{array}{c}
A \\
-I
\end{array}\right) x^{*} \geq \mathbf{0} .
$$

The point $x^{*}$ in (16) is $x^{*}=T y^{*}$. Notice that $x^{*}$ satisfies the system $A x \leq \alpha \mathbf{1}$.

Furthermore, we are interested in the facets of $Q_{I}$ which are not represented by the system $B y \leq d$. If $F$ is such a facet, then one can translate $y^{*}$ away from $Q_{I}$, without changing $y^{*}(n)=\beta+1 / 2$, such that $y^{*} \notin Q_{I}$ and $B y^{*} \leq d$ with the property that the facet we are considering is the unique inequality (12), where $f_{L}, f_{R}$ is a simple negative cycle in the graph $D=(U, \mathcal{A})$.

In the following we denote $U=\{1, \ldots, n\}$, where node $i$ corresponds to the $i$-th column of the matrix $M$ in (11). Notice that $\mathcal{A}$ partitions in two classes of arcs $\mathcal{A}_{L}$ and $\mathcal{A}_{R}$. The $\operatorname{arcs} \mathcal{A}_{R}$ are simply the reverse of the $\operatorname{arcs} \mathcal{A}_{L} . \mathcal{A}_{L}$ consists of two sets of $\operatorname{arcs} \mathcal{S}_{L}$ and $\mathcal{T}_{L}$, where $\mathcal{S}_{L}$ is the set of arcs associated with inequalities $A x \leq \alpha \mathbf{1}$ and $\mathcal{T}_{L}$ are the arcs stemming from the lower bounds $x \geq 0$. Likewise $\mathcal{A}_{R}$ can be partitioned into $\mathcal{S}_{R}$ and $\mathcal{T}_{R}$. In other words, if we look at the arc-node incidence matrix $M$ in (11), the rows of $M$ appear in the order $\mathcal{S}_{L}, \mathcal{T}_{L}, \mathcal{S}_{R}, \mathcal{T}_{R}$. 


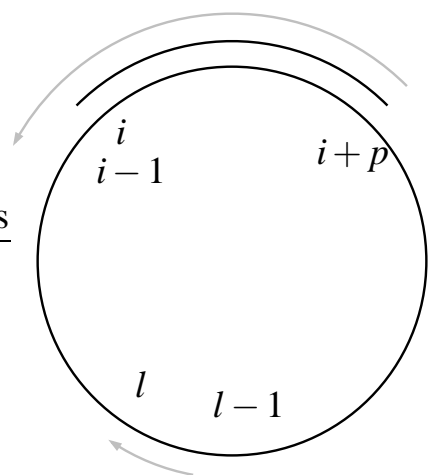

Figure 3: The incidence vector of a row of $A$ consists of the nodes $\{i, i+1, \ldots, i+p\}$ which are consecutive on the cycle in clockwise order. Its corresponding arc in $\mathcal{S}_{L}$ is the $\operatorname{arc}(i+p, i-1)$. The $\operatorname{arc}(l-1, l)$ in $\mathcal{T}_{L}$ corresponds to the lower bound $x(l) \geq 0$.

In particular, let $a$ denote a row vector of $A$. Since $A$ is a circular ones matrix one has $a x \leq \alpha \equiv$ $\sum_{h=0}^{p} x(i+h) \leq \alpha$ for some suitable $i$ and $p$, where computation is modulo $n$, so $x_{n} \equiv x_{0}, x_{n+1} \equiv x_{1}$, etc. It is straightforward to see that $a x \leq \alpha$ generates the arcs $(i+p, i-1) \in \mathcal{S}_{L}$ and $(i-1, i+p) \in \mathcal{S}_{R}$ of $\mathcal{A}$, see Figure 3. The weights of the two arcs coincide, if $n \notin\{i, i+1, \ldots, i+p\}$ and is exactly the slack $\alpha-\sum_{h=0}^{p} x^{*}(i+h)$ in this case. Otherwise, the weight of the $\operatorname{arc}(i+p, i-1)$ is $\alpha-\sum_{h=0}^{p} x^{*}(i+h)+1 / 2$ and the weight of the $\operatorname{arc}(i-1, i+p)$ is $\alpha-\sum_{h=0}^{p} x^{*}(i+h)-1 / 2$.

On the other hand, a lower bound $-x_{i} \leq 0$ generates the two $\operatorname{arcs}(i-1, i) \in \mathcal{T}_{L}$ and $(i, i-1) \in \mathcal{T}_{R}$. The weight of both $\operatorname{arcs}$ is equal to $x^{*}(i)$, if $i \neq n$. If $i=n$, the $\operatorname{arc}(n-1, n) \in \mathcal{T}_{L}$ has weight $x^{*}(n)-1 / 2$ and $(n, n-1) \in \mathcal{T}_{R}$ has weight $x^{*}(n)+1 / 2$.

Since the slacks are non-negative, the arcs whose cost is equal to the corresponding slack minus $\frac{1}{2}$ are the only candidates to have a negative cost. We call those light arcs. Consequently we call those arcs whose cost is equal to the slack plus $\frac{1}{2}$ heavy. Observe that the light arcs belong to $\mathcal{S}_{R} \cup\{(n-1, n)\}$.

Lemma 9. Let $\mathcal{C}$ be a simple negative cycle in $D$, then the following holds:

(a) C contains strictly more light arcs than heavy ones.

(b) An arc of $\mathcal{C}$ in $\mathcal{S}_{L}\left(\mathcal{T}_{L}\right)$ cannot be immediately followed or preceded by an arc in $\mathcal{S}_{R}\left(\mathcal{T}_{R}\right)$.

(c) The cycle $\mathcal{C}$ contains at least one arc of $\mathcal{S}_{R}$ or contains no arc of $\mathcal{S}_{L} \cup \mathcal{S}_{R}$.

Proof. (a) follows from the fact that the slacks are nonnegative. (b) follows from our assumption that the rows of the matrix $A$ are maximal and that $\mathcal{C}$ is simple.

To prove (c) suppose that the contrary holds. It follows that $(n-1, n)$ is in $\mathcal{C}$, because it is the only light arc not in $\mathcal{S}_{R}$. We must reach $n-1$ on the cycle without using heavy arcs.

Each $\operatorname{arc}$ in $\mathcal{S}_{L}$ with starting node $n$ is heavy. Thus $(n-1, n)$ is followed by $(n, 1) \in \mathcal{T}_{L}$. Suppose that $(n-1, n)$ is followed by a sequence of arcs in $\mathcal{T}_{L}$ leading to $i$ and let $(i, j) \notin \mathcal{T}_{L}$ be the arc which follows this sequence. It follows from (b) that $(i, j) \notin \mathcal{T}_{R}$ and thus that $(i, j) \in \mathcal{S}_{L}$. Since $(i, j)$ cannot be heavy, we have $1 \leq j<i<n$. This is a contradiction to the fact that $\mathcal{C}$ is simple, since we have a sub-cycle contained in $\mathrm{C}$, defined by $(i, j)$ and $(j, j+1), \ldots,(i-1, i)$.

Lemma 10. If there exists a simple cycle $\mathcal{C}$ of $D$ with negative cost, then there exists a simple cycle $\mathcal{C}^{\prime}$ of $D$ with negative cost that does not contain any arc from $S_{L}$. 
Proof. Suppose that $\mathcal{C}$ also contains an arc from the set $\mathcal{S}_{L}$. We know from Lemma 9 that the cycle $\mathcal{C}$ contains at least one arc of $\mathcal{S}_{R}$. Lemma 9 implies that $\mathcal{C}$ has an $\operatorname{arc}$ in $\mathcal{S}_{L}$, followed by $\operatorname{arcs}$ in $\mathcal{T}_{L}$ or $\mathcal{T}_{R}$ but not both, followed by an arc in $\mathcal{S}_{R}$. We first consider the case that the intermediate arcs are all in $\mathcal{T}_{L}$.

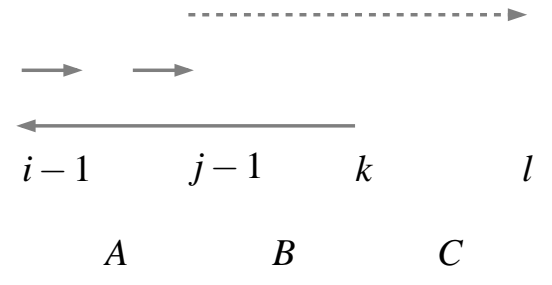

(a)

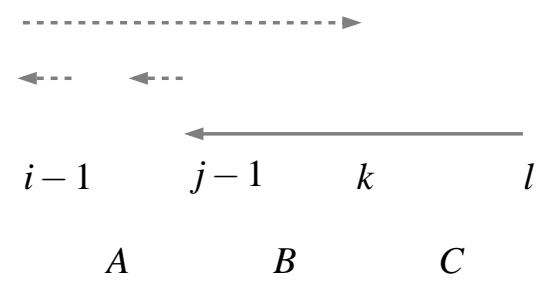

(b)

Figure 4: (a) depicts an $\operatorname{arc}(k, i-1) \in \mathcal{S}_{L}$, followed by $\operatorname{arcs}$ in $\mathcal{T}_{L}$ and the $\operatorname{arc}(j-1, l) \in \mathcal{S}_{R}$. (b) depicts the situation, where the intermediate arcs are in $\mathcal{T}_{R}$.

This situation is depicted in Figure 4, (a). The arc in $\mathcal{S}_{L}$ is $(k, i-1)$. This is followed by the arcs $(i-1, i), \ldots,(j-2, j-1)$ in $\mathcal{T}_{L}$ and the $\operatorname{arc}(j-1, l)$ in $\mathcal{S}_{R}$. Let this be the path $\mathcal{P}_{1}$. We now show that we can replace this path with the path $\mathcal{P}_{2}=(k, k+1), \ldots,(l-1, l)$ consisting of arcs in $\mathcal{T}_{L}$. We proceed as follows. First we show that the weight of this path is at most the weight of the original path, where we ignore the addition of $\pm 1 / 2$ to the arc-weights. Let $\operatorname{light}(\mathcal{P})$ and heavy $(\mathcal{P})$ be the number of light and heavy edges in a path $\mathcal{P}$, respectively. We then show that $\operatorname{light}\left(\mathcal{P}_{2}\right)-\operatorname{heavy}\left(\mathcal{P}_{2}\right)=\operatorname{light}\left(\mathcal{P}_{1}\right)-\operatorname{heavy}\left(\mathcal{P}_{1}\right)$, from which we can conclude the claim in this case.

Consider the set of indices $\mathcal{A}=\{i, \ldots, j-1\}, \mathcal{B}=\{j, \ldots, k\}$ and $\mathcal{C}=\{k+1, \ldots, l\}$ and the numbers $A=\sum_{\mu \in \mathcal{A}} x^{*}(\mu), B=\sum_{\mu \in \mathcal{B}} x^{*}(\mu)$ and $C=\sum_{\mu \in C^{*}} x^{*}(\mu)$. Ignoring the eventual addition of $\pm 1 / 2$ to the edge weights, we have that the weight of $\mathcal{P}_{2}$ is $C$ and that of $\mathcal{P}_{1}$ is $\alpha-(A+B)+A+\alpha-(B+C)$ and suppose that this is less than $C$. Then $B+C>\alpha$ which is not possible, since $x^{*}$ satisfies the constraints $A x \leq \alpha 1$. Thus, if none of the edges in $\mathcal{P}_{1}$ and $\mathcal{P}_{2}$ is heavy or light, the weight of $\mathcal{P}_{2}$ is at most the weight of $\mathcal{P}_{1}$.

Suppose now that $n \in \mathcal{A}$. Then $\mathcal{P}_{1}$ contains exactly one heavy edge $(k, i-1)$ and one light edge $(n-1, n)$. The path $\mathcal{P}_{2}$ contains no heavy or light edge. Suppose that $n \in \mathcal{B}$, then $\mathcal{P}_{1}$ contains exactly one heavy edge, $(k, i-1)$ and one light edge $(j-1, l) . \mathcal{P}_{2}$ does not contain a heavy or light edge. If $n \in C$, then $\mathcal{P}_{1}$ contains exactly one light edge $(j-1, l)$ and no heavy edge. $\mathcal{P}_{2}$ also contains exactly one light edge $(n-1, n)$. This concludes the claim for the case that an arc of $\mathcal{S}_{L}$ is followed by arcs of $\mathcal{T}_{L}$ and an $\operatorname{arc}$ of $S_{R}$.

The case, where the intermediate arcs belong to $\mathcal{T}_{R}$ is depicted in Figure 4, (b). The assertion follows by a similar argument.

Combining Theorem 7 with the above lemma we obtain the following theorem.

Theorem 11. Let $P=\left\{x \in \mathbb{R}^{n} \mid A x \leq \alpha \mathbf{1}, x \geq 0\right\}$ be a polyhedron, where $A \in\{0,1\}^{m \times n}$ is a circular ones matrix and $\alpha \in \mathbb{N}$ a positive integer. A facet of $P_{I}$ is of the form

$$
a \sum_{v \in T} x(v)+(a-1) \sum_{v \notin T} x(v) \leq a \beta,
$$

where $T \subseteq\{1, \ldots, n\}$ and $a, \beta \in \mathbb{N}$.

Proof. Theorem 7 implies that a facet which is not induced by $A x \leq \alpha \mathbf{1}, x \geq 0$ or $\mathbf{1} x \leq \gamma$ is a nonnegative integer combination of the system on the left in (14) with nonnegative weights $f_{L, 0}, f_{L}$. Lemma 10 implies 
that $f_{L}$ can be chosen such that the only nonzero $(+1)$ entries of $f_{L}$ are corresponding to lower bounds $-x(v) \leq 0$. The theorem thus follows with $a=f_{0, L}$ and $T$ set to those variables, whose lower bound inequality does not appear in the derivation.

\section{The solution of the Ben Rebea Conjecture}

Let $G$ be a circular interval graph and let $\mathcal{K}_{I}$ the family of cliques stemming from the intervals in the definition of $G$ (see Section 2). Then $P=\left\{x \in R^{n} \mid A x \leq \mathbf{1}, x \geq 0\right\}$ where the $0 / 1$ matrix $A$, corresponding to the cliques $\mathcal{K}_{I}$, has the circular ones property. Theorem 11 implies that any facet of $\operatorname{STAB}(G)$ is of the form

$$
a \sum_{v \in T} x(v)+(a-1) \sum_{v \notin T} x(v) \leq a \cdot \beta
$$

We now show that a facet $F$, which is not induced by an inequality of $A x \leq \mathbf{1}, x \geq 0$ is induced by a clique family inequality associated with some set of cliques $\mathcal{F} \subseteq \mathcal{K}_{I}$ and some integer $p$. Recall from Theorem 7 that any facet of this kind can be derived from the system

$$
\begin{aligned}
-\mathbf{1} x & \leq-(\beta+1) \\
A x & \leq \mathbf{1} \\
-x & \leq 0
\end{aligned}
$$

with weights $\left|f_{R, 0}\right|, f_{R}$, where $f_{R, 0}$ is a negative integer while $f_{R}$ is a $0-1$ vector. A root of $F$ is a stable set, whose characteristic vector belongs to $F$. In particular, we have that the multiplier $f_{R}(v)$ associated with a lower bound $-x(v) \leq 0$ must be 0 if $v$ belongs to a root of size $\beta+1$. If $v$ does not belong to a root of size $\beta$ or to a root of size $\beta+1$, then the facet is induced by $x(v) \geq 0$. Thus if $v \notin T$, then $v$ belongs to a root of size $\beta+1$.

Let $\mathcal{F}=\left\{K \in \mathcal{K}_{I} \mid f_{R}(K) \neq 0\right\}$ and $p=a+\left|f_{R, 0}\right|$. The multiplier $\left|f_{R, 0}\right|$ must satisfy

$$
\begin{array}{rlrl}
-\left|f_{R, 0}\right|+|\{K \in \mathcal{F} \mid v \in K\}| & =a-1 & & \forall v \notin T \\
-\left|f_{R, 0}\right|+|\{K \in \mathcal{F} \mid v \in K\}| & =a & & \forall v \in T, v \text { is in a root of size } \beta+1 \\
-\left|f_{R, 0}\right|+|\{K \in \mathcal{F} \mid v \in K\}| \geq & a & & \forall v \in T, v \text { is not in a root of size } \beta+1 \\
-\left|f_{R, 0}\right|(\beta+1)+|\mathcal{F}| & =a \beta &
\end{array}
$$

Observe that $|\mathcal{F}|=\left(a+\left|f_{R, 0}\right|\right) \beta+\left|f_{R, 0}\right|$ and therefore $r=|\mathcal{F}| \bmod p=\left|f_{R, 0}\right|$. Moreover, any vertex not in $T$ belongs to exactly $p-1$ cliques from $\mathcal{F}$, while each vertex in $T$ belongs to at least $p$ cliques from $\mathcal{F}$. Therefore, inequality (18) is the clique family inequality associated with $\mathcal{F}$ and $p$. In particular since $a \geq 1$ and $\left|f_{R, 0}\right| \geq 1$, it follows that $p \geq 2$. We may therefore state the following theorem.

Theorem 12. Let $G$ be a circular interval graph. Then any facet of $S T A B(G)$, which is not induced by an inequality of the system $A x \leq \mathbf{1}, x \geq 0$, is a clique family inequality associated with some $\mathcal{F}$ and $p$ such that $|\mathcal{F}| \bmod p \neq 0$.

If we combine this result with Lemma 5, Theorem 4 and we recall that Edmonds inequalities are also clique family inequalities associated with $|\mathcal{F}|$ odd and $p=2$, we obtain the following.

Theorem 13. Let $G$ be a quasi-line graph. Any non-trivial facet of $S T A B(G)$ is a clique family inequality associated with some $\mathcal{F}$ and $p$ such that $|\mathcal{F}| \bmod p \neq 0$.

The Ben Rebea conjecture is now almost settled. Inspecting it again, we observe that apart from the statement that the stable set polytope is described by nonnegativity, clique and clique family inequalities it contains also conditions on $\mathcal{F}$ and $p$. We may assume that the cliques in the family $\mathcal{F}$ are maximal [27]. What remains is the condition $|\mathcal{F}|>2 p \geq 4$. This is settled in the following, where we also show that clique family inequality are facet inducing only if $V_{\geq p} \neq \emptyset$. 
Lemma 14. A clique family inequality associated with $\mathcal{F}$ and $p$ is facet inducing only if $V_{\geq p} \neq \emptyset$.

Proof. If $V_{\geq p}=\emptyset$, then the clique family inequality associated with $\mathcal{F}$ and $p$ reads:

$$
(p-r-1) \sum_{v \in V_{p-1}} x(v) \leq(p-r)\left\lfloor\frac{n}{p}\right\rfloor
$$

We claim that this inequality is dominated by the clique family inequality associated with $\mathcal{F}$ and $p-1$ :

$$
\left(p-r^{\prime}-1\right) \sum_{v \in V_{\geq p-1}} x(v)+\left(p-r^{\prime}-2\right) \sum_{v \in V_{p-2}} x(v) \leq\left(p-1-r^{\prime}\right)\left\lfloor\frac{n}{p-1}\right\rfloor
$$

where $r^{\prime}=|\mathcal{F}| \bmod (p-1)$.

Since $p-r-1 \neq 0$ we can rewrite (20) as

$$
\sum_{v \in V_{p-1}} x(v) \leq\left\lfloor\frac{n}{p}\right\rfloor+\frac{\left\lfloor\frac{n}{p}\right\rfloor}{p-r-1} .
$$

Then, since $V_{p-1} \subseteq V_{\geq p-1}$, inequality (21) dominates:

$$
\sum_{v \in V_{p-1}} x(v) \leq\left\lfloor\frac{n}{p-1}\right\rfloor
$$

Now $\lfloor n /(p-1)\rfloor=\lfloor n / p\rfloor+\left\lfloor\left(\left\lfloor\frac{n}{p}\right\rfloor+r\right) /(p-1)\right\rfloor$ and the claim follows since

$$
\left\lfloor\frac{\left\lfloor\frac{n}{p}\right\rfloor+r}{p-1}\right\rfloor \leq \frac{\left\lfloor\frac{n}{p}\right\rfloor}{p-r-1} .
$$

Lemma 15. Let $G$ be a quasi-line graph and $(\mathcal{F}, p)$ a pair such that

$$
(p-r-1) \sum_{v \in V_{p-1}} x(v)+(p-r) \sum_{v \in V_{\geq p}} x(v) \leq(p-r)\left\lfloor\frac{|\mathcal{F}|}{p}\right\rfloor
$$

is a facet of $\operatorname{STAB}(G)$. If $|\mathcal{F}|<2 p$, then the inequality (22) is a clique inequality.

Proof. We know from the previous lemma that $V_{\geq p} \neq \emptyset$. Since $\left\lfloor\frac{|\mathcal{F}|}{p}\right\rfloor=1$, if $V_{p-1}=\emptyset$ or $p-r=1$, then the inequality (22) is a clique inequality, and we are done. Therefore we may assume that $V_{p-1} \neq \emptyset$ and $p-r>1$. Since the inequality is facet inducing, then $p-r=2$ and it reads:

$$
\sum_{v \in V_{p-1}} x(v)+2 \sum_{v \in V_{\geq p}} x(v) \leq 2
$$

Trivially, the inequality is also facet-inducing for the induced subgraph $G^{\prime}=G\left[V_{p-1} \cup V_{\geq p}\right]$. A full description of the stable set polytope of graphs with stability number less than three, as $G^{\prime}$, is given in [21]. There it is shown that an inequality $\sum_{v \in A} x(v)+2 \sum_{v \in B} x(v) \leq 2$, with $A$ and $B$ both non-empty, is facet inducing only if $B$ is a clique, $A$ and $B$ are totally joined and there is an odd antihole in $G[A]$. But no vertex of a quasi-line graph is totally joined to an odd antihole (from the definition of quasi-line graphs), so there is a contradiction.

We may therefore state our main result:

Theorem 16. The Ben Rebea conjecture holds true. 


\section{Acknowledgements}

We are in debt to Maria Chudnovsky for communicating Lemma 1 to us and, more in general, we are in debt to her and Paul Seymour for many useful discussions. A special thank is due to Adam Letchford for a very fruitful discussion about disjunctive cuts. We also thank Tom Liebling, Antonio Sassano, Bruce Shepherd, and Leslie Trotter for several useful discussions, support and encouragement.

\section{References}

[1] R. K. Ahuja, T. L. Magnanti, and J. B. Orlin, Network flows, Prentice Hall Inc., Englewood Cliffs, NJ, 1993, Theory, algorithms, and applications. MR 94e:90035

[2] K. Andersen, G. Cornuéjols, and Y. Li, Split closure and intersection cuts, Integer programming and combinatorial optimization, Lecture Notes in Comput. Sci., vol. 2337, Springer, Berlin, 2002, pp. 127-144. MR MR2061050

[3] J. J.III Bartholdi, J. B. Orlin, and H.D. Ratliff, Cyclic scheduling via integer programs with circular ones., Operations Research 28 (1980), 1074-1085.

[4] A. Caprara and A. N. Letchford, On the separation of split cuts and related inequalities, Mathematical Programming. A Publication of the Mathematical Programming Society 94 (2003), no. 2-3, Ser. B, 279-294, The Aussois 2000 Workshop in Combinatorial Optimization.

[5] M. Chudnovsky, Personal communication, 2004.

[6] M. Chudnovsky and P. Seymour, The structure of claw-free graphs, Proceedings of the Bristish Combinatorial Conference, Durham, 2005, to appear.

[7] V. Chvátal, Edmonds polytopes and a hierarchy of combinatorial problems, Discrete Mathematics 4 (1973), 305-337.

[8] V. Chvátal, W. Cook, and M. Hartmann, On cutting-plane proofs in combinatorial optimization, Linear Algebra and its Applications 114/115 (1989), 455-499.

[9] W. Cook, R. Kannan, and A. Schrijver, Chvátal closures for mixed integer programming problems, Mathematical Programming 47 (1990), 155-174.

[10] J. Edmonds, Maximum matching and a polyhedron with 0,1-vertices, Journal of Research of the National Bureau of Standards 69 (1965), 125-130.

[11] _ Paths, trees and flowers, Canadian Journal of Mathematics 17 (1965), 449-467.

[12] F. Eisenbrand, On the membership problem for the elementary closure of a polyhedron, Combinatorica 19 (1999), no. 2, 297-300.

[13] A. Galluccio and A. Sassano, The rank facets of the stable set polytope for claw-free graphs, Journal on Combinatorial Theory 69 (1997), 1-38.

[14] D. Gijswijt, Integer decomposition for polyhedra defined by nearly totally unimodular matrices, to appear in Siam Journal on Discrete Mathematics, 2005.

[15] R. Giles and L. E. Trotter, Jr., On stable set polyhedra for $K_{1,3}$-free graphs, J. Combin. Theory Ser. B 31 (1981), no. 3, 313-326. MR MR638287 (84a:05041)

[16] R. E. Gomory, Outline of an algorithm for integer solutions to linear programs, Bulletin of the American Mathematical Society 64 (1958), 275-278.

[17] M. Grötschel, L. Lovász, and A. Schrijver, The ellipsoid method and its consequences in combinatorial optimization, Combinatorica 1 (1981), no. 2, 169-197.

[18] M. Grötschel, L. Lovász, and A. Schrijver, Geometric algorithms and combinatorial optimization, Springer Verlag, Berlin, 1988.

[19] R. M. Karp and C. H. Papadimitriou, On linear characterizations of combinatorial optimization problems, SIAM Journal on Computing 11 (1982), no. 4, 620-632.

[20] L.G. Khachiyan, A polynomial algorithm in linear programming, Doklady Akademii Nauk SSSR 244 (1979), 1093-1097.

[21] T. M. Liebling, G. Oriolo, B. Spille, and G. Stauffer, On the non-rank facets of the stable set polytope of claw-free graphs and circulant graphs, Mathematical Methods of Operations Research 59 (2004), $25-35$.

[22] L. Lovász and M.D. Plummer, Matching theory, North Holland, Amsterdam, 1986. 
[23] J.-F. Maurras, Convex hull of the edges of a graph and near bipartite graphs, Discrete Math. 46 (1983), no. 3, 257-265. MR MR716446 (84m:05070)

[24] G. J. Minty, On maximal independent sets of vertices in claw-free graphs, Journal on Combinatorial Theory 28 (1980), 284-304.

[25] D. Nakamura and A. Tamura, A revision of Minty's algorithm for finding a maximum weighted stable set of a claw-free graph, Journal of the Operations Research Society of Japan 44 (2001), no. 2, 194-2004.

[26] G. L. Nemhauser and L. A. Wolsey, Integer and combinatorial optimization, John Wiley, 1988.

[27] G. Oriolo, Clique family inequalities for the stable set polytope for quasi-line graphs, Discrete Applied Mathematics 132 (2003), no. 3, 185-201.

[28] M. W. Padberg and M. R. Rao, The Russian method for linear programming III: Bounded integer programming, Tech. Report 81-39, New York University, Graduate School of Business and Administration, 1981.

[29] W. R. Pulleyblank and F. B. Shepherd, Formulations for the stable set polytope of a claw-free graph, Proceedings Third IPCO Conference (G. Rinaldi and L. Wolsey, eds.), 1993, pp. 267-279.

[30] A. Ben Rebea, Étude des stables dans les graphes quasi-adjoints, Ph.D. thesis, Université de Grenoble, 1981.

[31] N. Sbihi, Algorithme de recherche d'un stable de cardinalité maximum dans un graphe sans étoile, Discrete Mathematics 29 (1980), 53-76.

[32] A. Schrijver, On cutting planes, Annals of Discrete Mathematics 9 (1980), 291-296.

[33] _ Theory of linear and integer programming, John Wiley, 1986.

[34] A. Schrijver, Combinatorial optimization. Polyhedra and efficiency (3 volumes)., Algorithms and Combinatorics 24. Berlin: Springer., 2003.

[35] F. B. Shepherd, Applying Lehman's theorems to packing problems, Mathematical Programming 71 (1995), 353-367. 